\title{
Adaptive State Predictor Based Human Operator Modeling on Longitudinal and Lateral Control
}

\author{
Anna C. Trujillo* and Irene M. Gregory, Ph.D. ${ }^{\dagger}$ \\ NASA Langley Research Center, Hampton, VA, 23681, USA \\ Lucas E. Hempley ${ }^{\ddagger}$ \\ Northrop Grumman, Hampton, VA, 23681, USA
}

\begin{abstract}
Control-theoretic modeling of the human operator dynamic behavior in manual control tasks has a long and rich history. In the last two decades, there has been a renewed interest in modeling the human operator. There has also been significant work on techniques used to identify the pilot model of a given structure. The purpose of this research is to attempt to go beyond pilot identification based on collected experimental data and to develop a predictor of pilot behavior. An experiment was conducted to categorize these interactions of the pilot with an adaptive controller compensating during control surface failures. A general linear in-parameter model structure is used to represent a pilot. Three different estimation methods are explored. A gradient descent estimator (GDE), a least squares estimator with exponential forgetting (LSEEF), and a least squares estimator with bounded gain forgetting (LSEBGF) used the experiment data to predict pilot stick input. Previous results have found that the GDE and LSEEF methods are fairly accurate in predicting longitudinal stick input from commanded pitch. This paper discusses the accuracy of each of the three methods - GDE, LSEEF, and LSEBGF - to predict both pilot longitudinal and lateral stick input from the flight director's commanded pitch and bank attitudes.
\end{abstract}

\section{Introduction}

Significant research has been conducted to model or identify the pilot, as a way to quantify handling Squalities or to better understand the behavior of a human pilot in controlling a vehicle. ${ }^{1-7}$ The classical McRuer crossover model of compensatory manual control states that the human operator will adapt to vehicle dynamics by providing lead equalization, with an associated computational penalty, such that the combined operator-vehicle transfer function is proportional to an integrator at the crossover frequency. ${ }^{7}$ Another approach to understanding the human-as-the-controller behavior was the development of the optimal control model by Kleinman, Baron, and Levinson based on the assumption that a well-trained and motivated human controller behaves optimally in some sense, adjusting the pilot's compensation for a given vehicle and task, subject to human limitations. ${ }^{8}$

In the last two decades, there has been a renewed interest in modeling the human operator. Further model development by Hess in the frequency domain, a structural model,${ }^{9}$ and Schmidt in the time domain, a modified optimal control pilot model, ${ }^{2,10}$ attempted to take into consideration appropriate relevant feedback that influenced a pilot's behavior such as proprioceptive cues, and visual and vestibular feedback. While these models proved useful, their application to realistic simulation tasks involved a great deal of complexity. In an attempt to simplify the model while still capturing the essential pilot behavioral characteristics, Hess proposed a simplified model that has been recently applied to systems with time-varying dynamics. ${ }^{11,12}$

In addition to several relevant model structures to describe the pilot-as-a-controller behavior, there has also been significant work on techniques used to identify the pilot model of a given structure. ${ }^{13-19}$ These

*Senior Research Engineer, Crew Systems and Aviation Operations Branch, MS 152, AIAA Member.

†Senior Research Engineer, Langley Flight Systems Lead, MS 308, AIAA Associate Fellow.

‡Engineer, Northrop Grumman Technical Services, MS 152. 
included various applications of parameter identification ranging from wavelets-based approach to frequencybased system identification techniques.

This research is investigating new analytical methods to model the pilots changing behavior over short time periods in response to changing aircraft dynamics. This research is attempting to go beyond pilot identification based on collected experimental data and to develop a predictor of short-term pilot control behavior in real time. The long term goal of this research is to describe a model that goes beyond identifying pilot behavior changes due to vehicle dynamic changes but captures changes in pilot's behavior due to a myriad of factors, such as pilot incapacitation. This may enable identification of potential reasons for such behavior change.

This paper is organized as follows: section II describes the experiment, section III gives a brief overview of the estimation methods used, section IV discusses the results, and section V offers conclusions.

\section{Adaptive Controller / Pilot Interaction Experiment}

The data this research used is from an experiment that was conducted to categorize the interaction of a pilot with an adaptive controller during control surface failures. This experiment is described below.

The adaptive controller / pilot interaction experiment was conducted to categorize the interactions between a pilot and an adaptive controller during a change in aircraft dynamics represented by control surface failures. ${ }^{20-22}$ One of the objectives of this experiment was to determine how the adaptation time of the controller affects pilots. This was accomplished by considering whether an adaptive controller helps pilots during control surface failures (by measuring tracking error) and how this controller affects pilots before, during, and after the control surface failures. The control surface failures were either stuck surfaces or slowly moving surfaces. A human-in-the-loop experiment looked at the effects of these control surface failures on pilot performance during the cruise phase while initiating a climb, descent, or a heading change maneuver. These maneuvers were indicated on the primary flight display (PFD) via the flight director and on the engine instrument displays (EID) horizontal and vertical navigation map displays. Each subject experienced four adaptation times: zero seconds, three seconds, seven seconds, and no adaptation (Never). These times indicated how long it took the adaptive controller-aircraft system to settle to a new set of dynamics and specific adaptation values are based on the response speed of the nominal aircraft dynamics. Zero seconds indicated the fastest possible adaptation time, essentially the processor speed. For a detailed description of this experiment, see Trujillo and Gregory ${ }^{20,21}$ and Trujillo, et al. ${ }^{22}$

\section{A. Independent Variables}

The above experiment supplied the data to determine the accuracy of the estimation algorithms in predicting subject stick input and the estimation algorithms' parameter values (estimation gain, forgetting rate, and maximum forgetting rate described in Eqs. 7-9 on page 4).

The primary independent variables were vehicle commanded pitch angle and vehicle commanded bank angle. Subjects saw this information displayed on the PFD. The difference in ability to predict longitudinal and lateral stick was also considered, thus making stick direction an independent variable.

Subject was also included as an independent variable because it was hypothesized that there might be a subject dependency. The above experiment collected flight technical data from 17 subjects, all of which were airline transport pilots.

The above experiment also controlled for time for the adaptive controller to fully adapt. Therefore, adaptation time (0 seconds (sec), $3 \mathrm{sec}, 7 \mathrm{sec}$, and Never) was also an independent variable.

Another independent variable, section, consisted of before, during, and after the failure adaptation. For an adaptation time of $0 \mathrm{sec}$, there is no during failure section and for an adaptation time of Never, there is no after failure section.

Table 1 on page 3 details these independent variables.

\section{B. Dependent Variables}

The estimation methods' parameter values (PV) are calculated using Eqs. 7-9 below (see Section III). These values are the estimation gain from the gradient descent estimator (Eq. 7), the forgetting rate from the least squares estimator with exponential forgetting (Eq. 8), and the gain matrix magnitude from the least squares estimator with bounded gain forgetting (Eq. 9). The forgetting rate, $\lambda$, was normalized to a range of 0-1. 
Table 1. Independent and Dependent Variables

\begin{tabular}{|c|c|}
\hline \multicolumn{2}{|r|}{ Independent Variables } \\
\hline Name & \begin{tabular}{|r} 
Values \\
Valu
\end{tabular} \\
\hline \multirow[t]{3}{*}{ Algorithm } & GDE \\
\hline & LSEEF \\
\hline & LSEBGF \\
\hline Subject & $1-17$ \\
\hline \multirow[t]{4}{*}{ Adaptation Time } & $0 \mathrm{sec}$ \\
\hline & $3 \mathrm{sec}$ \\
\hline & $7 \mathrm{sec}$ \\
\hline & Never \\
\hline \multirow[t]{3}{*}{ Section } & Before \\
\hline & During \\
\hline & After \\
\hline \multirow[t]{3}{*}{ Direction } & Lateral (Commanded Bank Angle) \\
\hline & Longitudinal (Commanded Pitch Angle) \\
\hline & Dependent Variables \\
\hline Name & Values \\
\hline \multirow[t]{3}{*}{ Parameter Value (PV) } & Estimation Gain $\left(\Gamma_{\Theta}\right): 1-100$ \\
\hline & Forgetting Rate $(\lambda): 0.01-1.00$ \\
\hline & Gain Matrix Magnitude $\left(k_{0}\right): 1-100$ \\
\hline $\mathrm{PV}_{d b}$ & $\mathrm{PV}_{\text {during }}-\mathrm{PV}_{\text {before }}$ \\
\hline $\mathrm{PV}_{a b}$ & $\mathrm{PV}_{\text {after }}-\mathrm{PV}_{\text {before }}$ \\
\hline |Average Error $\mid$ & $0-2$ \\
\hline $\mid$ Average Error $\left.\right|_{d b}$ & $\mid$ Average Error $\left.\right|_{\text {during }}-\mid$ Average Error $\left.\right|_{\text {before }}$ \\
\hline $\mid$ Average Error $\left.\right|_{a b}$ & $\mid$ Average Error $\left.\right|_{\text {after }}-\mid$ Average Error $\left.\right|_{\text {before }}$ \\
\hline
\end{tabular}

In order to determine the accuracy of the estimation methods, the predicted pilot longitudinal and lateral stick input was compared to actual subject longitudinal and lateral stick input collected from the experiment described above. Therefore,

$$
\mid \text { Average Error } \mid=\frac{\sum_{t=0}^{120} \mid \text { Predicted Stick Input }(t)-\text { Actual Stick Input }(t) \mid}{t}
$$

where $t$ is the data run time (in seconds). |Average Error| was calculated for each run for each subject. The stick inputs were calculated for both the lateral and longitudinal directions. Stick movement was normalized from -1 to +1 .

Lastly, to see whether there was a discernible difference in the parameter values or the predicted stick accuracies during the failure and after the failure as compared to before failure values, these were computed in the following manner:

$$
\begin{aligned}
& \mathrm{PV}_{d b}=\mathrm{PV}_{\text {during }}-\mathrm{PV}_{\text {before }} \\
& \mathrm{PV}_{d b}=\mathrm{PV}_{\text {during }}-\mathrm{PV}_{\text {before }}
\end{aligned}
$$

Table 1 also details these dependent variables. 


\section{Estimation Methods}

This section describes the three estimation methods. The algorithms to estimate pilot longitudinal stick input used vehicle commanded pitch attitude data from the experiment described above. The subjects' longitudinal and lateral stick inputs, and longitudinal and lateral flight director commands (commanded pitch angle and commanded bank angle respectively) were recorded in the above experiment (section II).

Consider a pilot input-output model given by a general linear in-parameter form

$$
y(t)=\Phi(x(t))^{T} \Theta(t)
$$

where $y(t)$ is system output (longitudinal or lateral stick in this instance), $x(t)$ is input signal (commanded pitch or bank angle in this instance), $\Phi(x(t))$ is the regressor composed of basis functions, and $\Theta(t)$ is an unknown parameter vector.

By estimating the unknown parameter vector, $\Theta(t)$, system output is estimated by Eq. 6 . Three different methods for parameter estimation are considered in building a pilot input-output model. These models are a gradient descent estimator (GDE), a least squares estimator with exponential forgetting (LSEEF), and a least squares estimator with bounded gain forgetting (LSEBGF). ${ }^{23}$

The online implementation of the GDE is given by

$$
\dot{\hat{\Theta}}(t)=-\Gamma \Phi(x(t))(\underbrace{\Phi(x(t))^{T} \hat{\Theta}(t)}_{\hat{y}(t)}-y(t))
$$

where $\Gamma$ is the estimation gain. Note longitudinal and lateral stick deflection was normalized to \pm 1 range.

Similarly, the online representation of the LSEEF is

$$
\begin{aligned}
\dot{\hat{\Theta}}(t) & =-\Gamma(t) \Phi(x(t))\left(\Phi(x(t))^{T} \hat{\Theta}(t)-y(t)\right), & \hat{\Theta}(0) & =\hat{\Theta}_{0} \\
\dot{\Gamma}(t) & =\lambda(t) \Gamma(t)-\Gamma(t) \Phi(x(t))^{T} \Gamma(t), & \Gamma(0) & =\Gamma_{0}
\end{aligned} .
$$

The variable $\lambda$, the forgetting rate, is part of exponential forgetting factor which enables LSEEF to track time-varying parameters, albeit slowly varying. The rest of the variables are the same as described above for GDE.

Lastly, the LSEBGF online representation is

$$
\begin{array}{rlrl}
\dot{\hat{\Theta}}(t) & =-\Gamma(t) \Phi(x(t))\left(\Phi(x(t))^{T} \hat{\Theta}(t)-y(t)\right), & \hat{\Theta}(0)=\hat{\Theta}_{0} \\
\dot{\Gamma}(t) & =\lambda(t) \Gamma(t)-\Gamma(t) \Phi(x(t))^{T} \Gamma(t), & \Gamma(0)=\Gamma_{0} \\
\lambda(t) & =\lambda_{0}\left(1-\frac{\|\Gamma(t)\|}{k_{0}}\right) & &
\end{array}
$$

where $\lambda_{0}$ is the maximum forgetting rate and $k_{0}$ is the pre-specified bound for gain matrix magnitude. The rest of the variables are the same as described above for LSEEF.

For GDE, the estimation gain, $\Gamma$, was recursively calculated for each subject's run until the estimated longitudinal and lateral stick inputs matched the actual longitudinal and lateral stick inputs as close as reasonably possible. The forgetting rate, $\lambda$, for LSEEF, was also recursively calculated for each subject's run until the estimated longitudinal and lateral stick inputs matched the actual stick inputs as close as reasonably possible. Finally, for the LSEBGF algorithm, the bound for gain matrix magnitude, $k_{0}$, as recursively calculated until a value of $\lambda$ was arrived at that the estimated longitudinal and lateral stick inputs reasonably matched the actual stick inputs - once again for each subject.

\section{Results}

Previous results have found that the GDE and LSEEF methods are fairly accurate in predicting longitudinal stick input from commanded pitch. ${ }^{24,25}$ These results will discuss the accuracy of each of the three methods - GDE, LSEEF, and LSEBGF - to predict both pilot longitudinal and lateral stick input from the flight director's commanded pitch and bank attitudes. The results will also discuss how $\Gamma, \lambda$, and $k_{0}$ vary 
depending on controller adaptation time during each run's three segments: before failure, during failure, and after failure.

The above estimators were implemented in MATLAB ${ }^{\circledR}$ and Simulink ${ }^{\circledR}$ R2013b ${ }^{\text {a }}$. Further data analyses was done using IBM SPSS ${ }^{\circledR}$ Statistics 22 . Significance was set at $p \leq 0.05$.

\section{A. LSEBGF $\lambda_{0}$ Variance}

As shown above in Eq. 9, $\lambda(t)$ is dependent on $\lambda_{0}$, the maximum forgetting rate. This value, $\lambda_{0}$, was initially set at the average LSEEF $\lambda(t)$ calculated from Eq. 8 before the failure occurred, which was 0.49 . Further analyses indicated that LSEEF $\lambda(t)$ varied by the direction independent variable, lateral and longitudinal, before the failure occurred $\left(F_{(1,1963)}=12.96 ; p \leq 0.01\right)$. Table 2 indicates the values $\lambda_{0}$ was set at when running the LSEBGF algorithm again when taking into consideration whether the lateral or longitudinal stick inputs were being computed.

However, when analyzing PV,PV $[d b, a b], \mid$ Average Error| and $\mid$ Average Error $\left.\right|_{[d b, a b]}$, the results for LSEBGF with $\lambda_{0}$ set to 0.49 irrespective of stick direction and for $\lambda_{0}$ set to 0.52 for the longitudinal direction and 0.46 for the lateral direction always grouped together when looking at the Tukey Honestly Significance Difference (HSD) post-hoc analysis. Therefore, further results using the LSEBGF algorithm will only be reported using $\lambda_{0}=0.49$.

\section{B. Subject Effects}

Not surprisingly, subject was statistically significant for PV and |Average Error|. However, when looking at the Tukey HSD post-hoc analysis, there was considerable overlapping in the groups. In general, once the overlapping was taken into consideration, subjects appeared to group into two groups - high and low. Table 3 details the differences between these two groups. Because the subjects could generally be split up into two groups, subject was included in the analysis model for PV and |Average Error| but subject and any interactions including subject will be reported using only the two subject groups.

Table 3. Subject Grouping

\begin{tabular}{|c|c|c|c|c|c|c|}
\hline \multirow[b]{2}{*}{ Section } & \multirow[b]{2}{*}{ Method } & \multirow[b]{2}{*}{ Direction } & \multicolumn{2}{|c|}{ PV } & \multicolumn{2}{|c|}{ |Average Error $\mid$} \\
\hline & & & $\begin{array}{c}\text { Group } 1 \\
\text { Value }\end{array}$ & $\begin{array}{c}\text { Group } 2 \\
\text { Value }\end{array}$ & $\begin{array}{c}\text { Group } 1 \\
\text { Value }\end{array}$ & $\begin{array}{c}\text { Group } 2 \\
\text { Value }\end{array}$ \\
\hline \multirow[t]{3}{*}{ Before } & GDE & & 10 & 15 & 0.0087 & 0.0094 \\
\hline & LSEEF & & 0.45 & 0.53 & 0.0311 & 0.0515 \\
\hline & LSEBGF & & 33 & 39 & 0.0528 & 0.0757 \\
\hline \multirow[t]{3}{*}{ During } & GDE & & 18 & 23 & 0.0271 & 0.0335 \\
\hline & LSEEF & & 0.37 & 0.47 & 0.0766 & 0.1016 \\
\hline & LSEBGF & & 27 & 35 & 0.1354 & 0.1750 \\
\hline \multirow[t]{3}{*}{ After } & GDE & & 17 & 22 & 0.0169 & 0.0226 \\
\hline & LSEEF & & 0.10 & 0.10 & 0.0664 & 0.1124 \\
\hline & LSEBGF & & 15 & 18 & 0.1543 & 0.2188 \\
\hline
\end{tabular}

When looking at the difference between before and during, and before and after the failure, subject was once again significant but the groupings from the Tukey HSD post-hoc analysis essentially collapsed into a single group. Therefore, subject was included in the analysis model for $\mathrm{PV}_{[d b, a b]}$ and $\mid$ Average Error $\left.\right|_{[d b, a b]}$ but subject and any interactions including subject will not be reported on.

\section{Ability of Estimation Methods to a Bounded Solution}

The three algorithms were able to predict subject stick input with varying degrees of success. GDE and LSEBGF were able to converge to a solution $86 \%$ and $76 \%$ of the time respectively while LSEEF only was

\footnotetext{
${ }^{a}$ The use of trademarks or names of manufacturers in the report is for accurate reporting and does not constitute an official endorsement, either expressed or implied, of such products or manufacturers by the National Aeronautics and Space Administration.
} 
able to converge to a solution $44 \%$ of the time. Convergence was defined as having the predicted output within $10 \%$ of the actual output over a defined sampling time (section in this case) with a local minimum for these differences. The success of the algorithms might have depended on the length of the runs, in this case $120 \mathrm{sec}$, and the amount of stick movement during this time, which was not a great deal considering most of the $120 \mathrm{sec}$ run was in straight and level flight. Longer sampling times with more stick movement may increase the success of the algorithms to converge to a solution. Irrespective of sampling time, the GDE and LSEBGF appear to be the best algorithms to use to converge to a solution.

\section{Accuracy of Estimation Methods}

The three estimation algorithms had varying degrees of ability to accurately predict subject stick input. This was hinted at in Table 3 on page 5 when looking at the |Average Error| columns. Illustrative examples of the algorithms' ability to predict actual stick commands are shown in Figure 1.
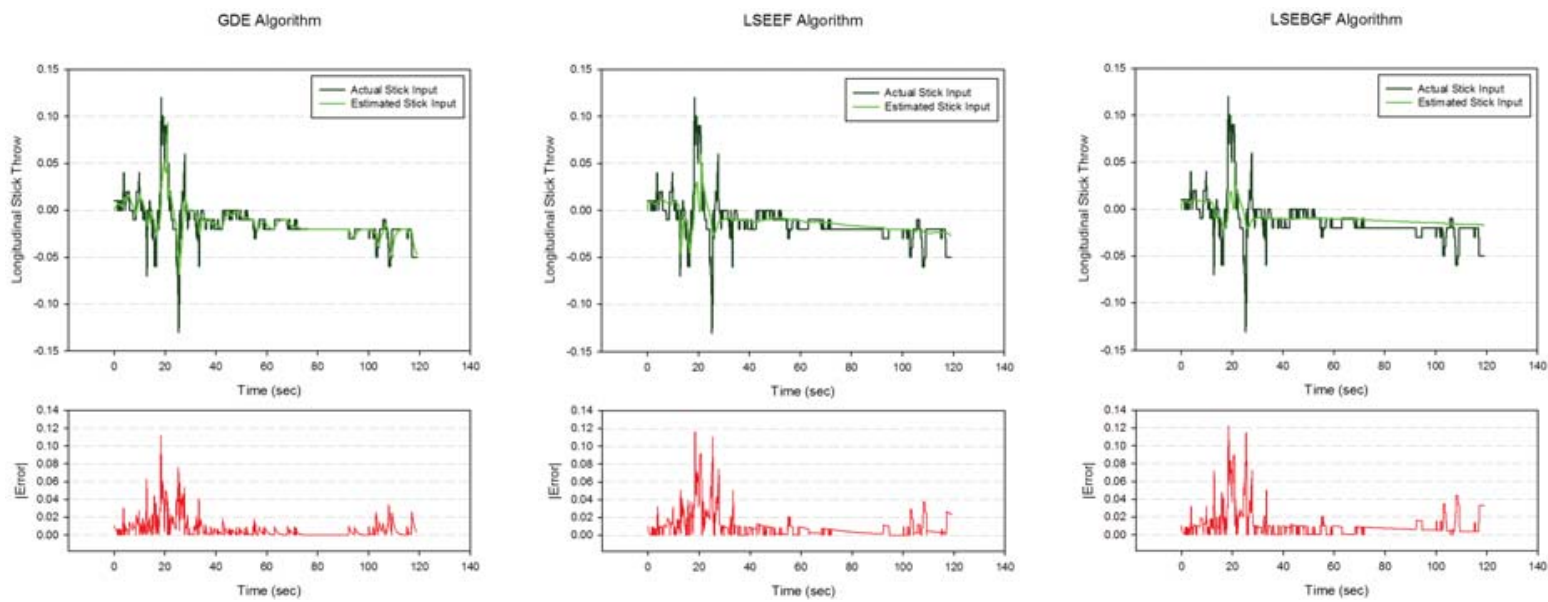

(a) GDE Algorithm Longitudinal Stick Estimation Example

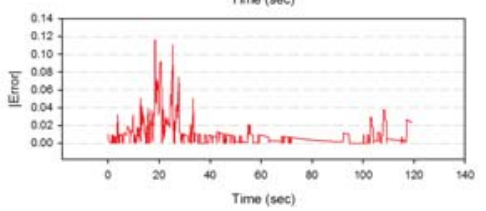

(b) LSEEF Algorithm Longitudinal Stick Estimation Example (c) LSEBGF Algorithm Longitudinal Stick Estimation Example

Figure 1. Representative Longitudinal Stick Estimation Examples with Absolute Error Between Actual and Estimated Stick Input

\section{Predicted Stick Input Accuracy Before Failure}

In the short time in the data runs before any failures occurred $(\leq 30 \mathrm{sec})$, subject by algorithm $\left(F_{(48,10858)}=\right.$ $1.72 ; p \leq 0.01)$ and subject by direction $\left(F_{(16,10858)}=2.16 ; p \leq 0.01\right)$ were significant for $\mid$ Average Error $\mid$. In general, the GDE algorithm was the most accurate in predicting stick input before the failure (Figure $2(\mathrm{a})^{\mathrm{b}}$ on page 7) irrespective of subject. In particular, the GDE method and lateral direction were the least sensitive to subject effects (Figures 2(a) and 2(b) respectively on page 7). Thus, if the PV is not specifically tuned to the pilot, then the GDE algorithm in the lateral direction provides the most accuracy in predicting pilot stick input during normal flight operations; and therefore, the GDE algorithm may be sensitive to detecting possible piloting changes during non-normal flight operations.

\section{Predicted Stick Input Accuracy During and After Failure}

As with the predicted stick input accuracy before the failure, the GDE algorithm was generally the most accurate, especially during the failure and for lateral stick input prediction (Figures 3(c) and 3(a) respectively on 7). Algorithm with adaptation time and direction $\left(F_{(9,10184)}=4.65, p \leq 0.01\right)$, and with adaptation time and section $\left(F_{(3,10184)}=3.46, p \leq 0.02\right)$ (Figure 3 on page 7$)$ were statistically significant. However, the GDE algorithm was not the most accurate in predicting longitudinal stick input and after the failure. In this case, the LSEBGF method was the most accurate (Figures 3(b) and 3(d) on page 7), albeit not by much. This was most likely due to the GDE method having a larger range of absolute average error for some of the data runs in the longitudinal direction and after the failure occurred. The errors in the longitudinal direction may

\footnotetext{
${ }^{\mathrm{b}}$ The error bars in the graphs indicate one standard error of the mean.
} 


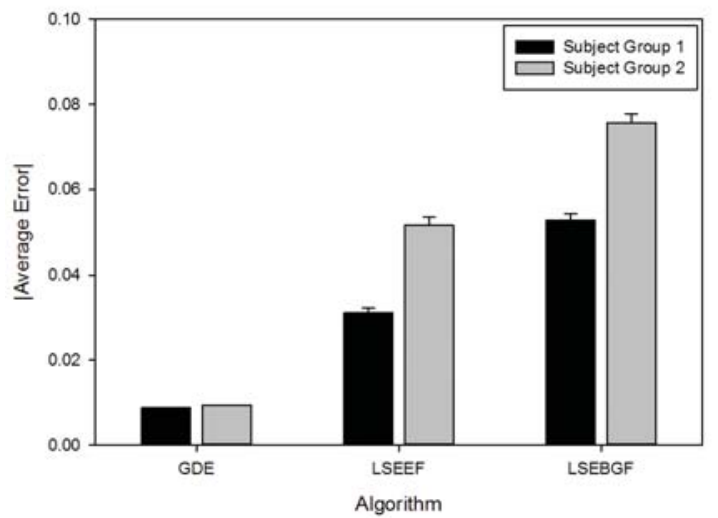

(a) Absolute Average Error by Algorithm and Subject Group

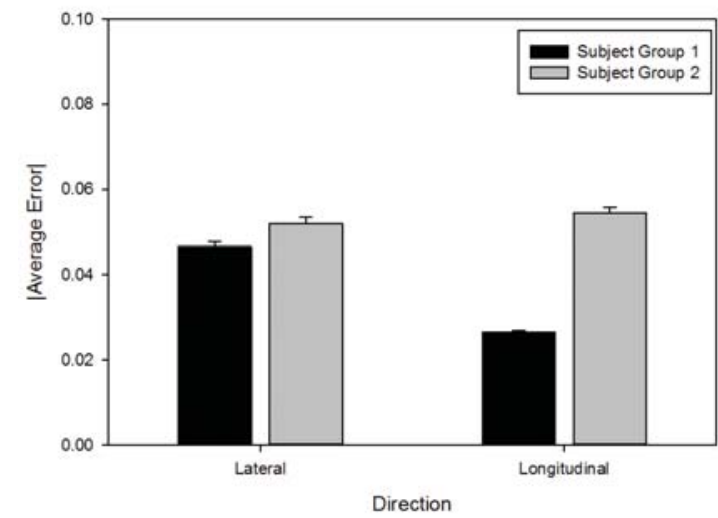

(b) Absolute Average Error by Algorithm and Subject Group

Figure 2. Predicted Stick Input Accuracy Before Failure

Lateral Direction

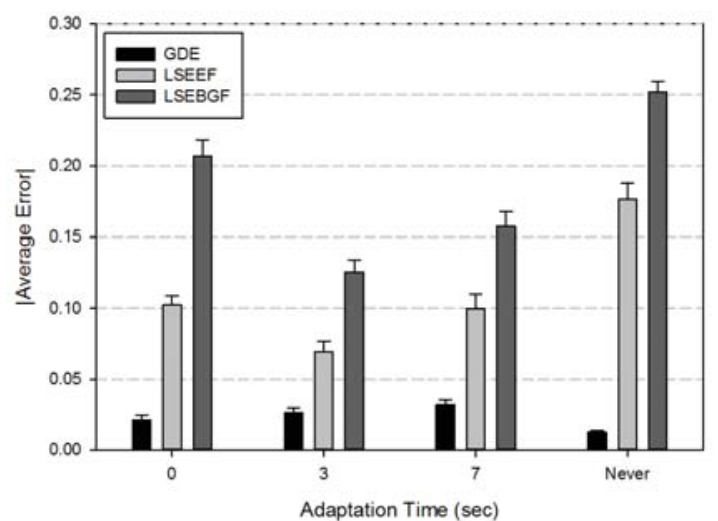

(a) Absolute Average Error by Algorithm and Adaptation Time for Lateral Direction

During Failure

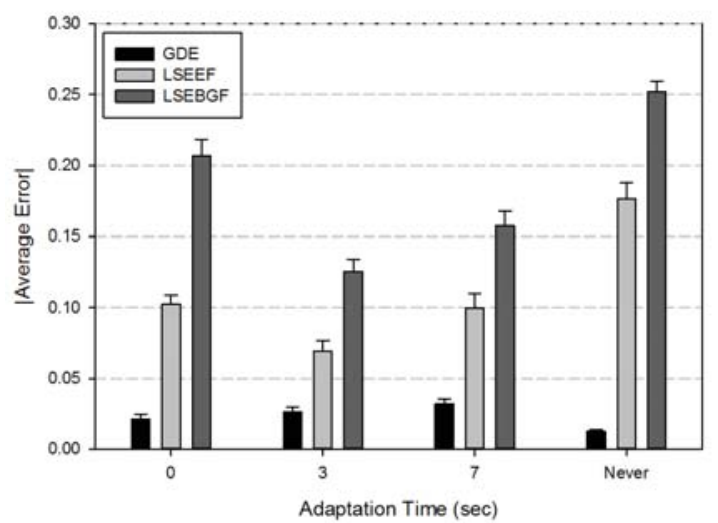

(c) Absolute Average Error by Algorithm and Adaptation Time During Failure
Longitudinal Direction

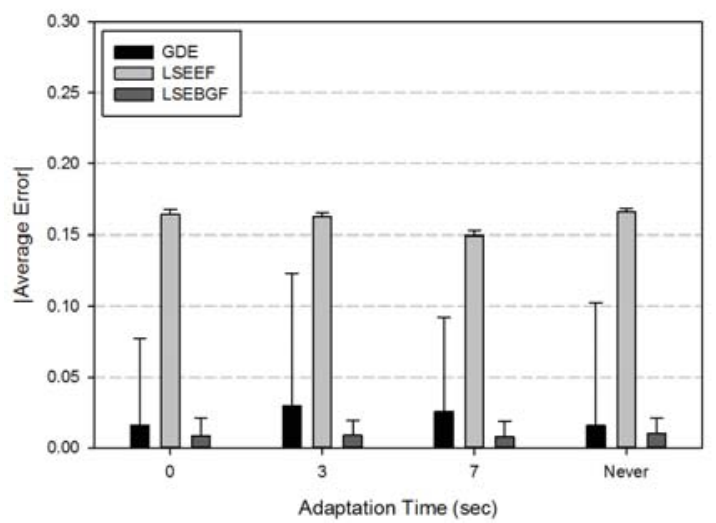

(b) Absolute Average Error by Algorithm and Adaptation Time for Longitudinal Direction

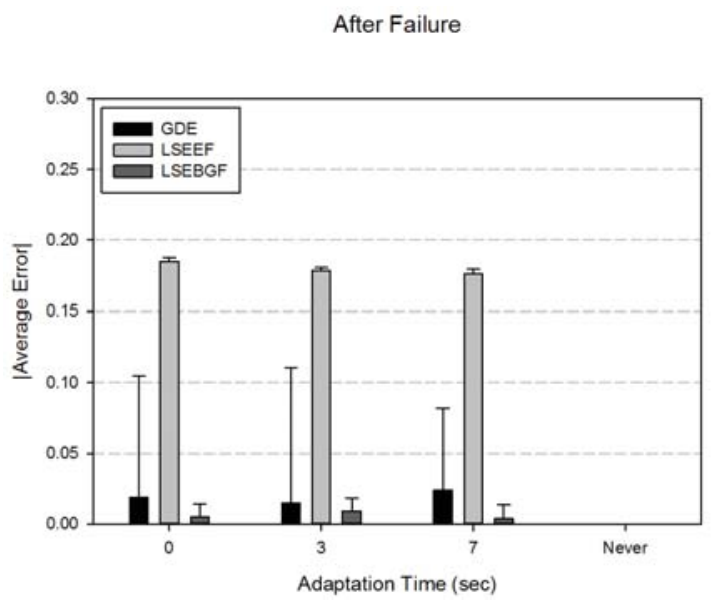

(d) Absolute Average Error by Algorithm and Adaptation Time After Failure

Figure 3. Algorithm Effects on Predicted Stick Input Accuracy 
be due to the data runs involving an altitude change which persisted for the rest of the data run after the altitude change was initiated; therefore, subjects had to deal with the failure and with the altitude change for the vast majority of the run. While subjects were constantly adjusting in the longitudinal direction for altitude changes, the errors may have been building up in the GDE algorithm whereas the LSEBGF algorithm with bounded gain forgetting may have been able to null out earlier large errors. If this is the case, then for detecting piloting changes in short runs, the GDE algorithm is still viable; however, for longer runs or where there are constant changes in flight path, then LSEBGF may be a better algorithm to estimate pilot stick input and to detect changes in piloting because of its forgetting characteristic.

\section{E. Ability to Detect Changes}

When trying to detect changes in piloting, subject was not significant when analyzing $\mathrm{PV}_{[d b, a b]}$ and $\mid$ Average Error $\left.\right|_{[d b, a b]}$. Therefore, it may be easier to detect changes in piloting behavior when looking at the differences between baseline (in this case, before failure) and later in time (in this case, during and after the failure) because individual differences do not play as an important a role.

\section{Absolute Average Error Difference Between Sections}

When looking at the absolute average error difference between before, and during and after sections, direction by section and adaptation time was significant $\left(F_{(1,5775)}=7.14, p \leq 0.01\right)$. As seen in Figure 4, it appears to be easier to detect changes in the longitudinal direction. When looking at the algorithm by direction interaction $\left(F_{(3,5775)}=4.56, p \leq 0.01\right)$ (Figure $5(\mathrm{a})$ on page 9 ) and the algorithm by section interaction $\left(F_{(3,5775)}=20.2, p \leq 0.01\right)$ (Figure 5(b) on page 9), the longitudinal direction and after - before section show changes in piloting behavior the most with the LSEBGF algorithm. For the GDE algorithm, the direction and section effects are minimal. Therefore, the LSEBGF algorithm may be best for detecting changes in piloting behavior when looking at the absolute average error differences between sections in the longitudinal direction.

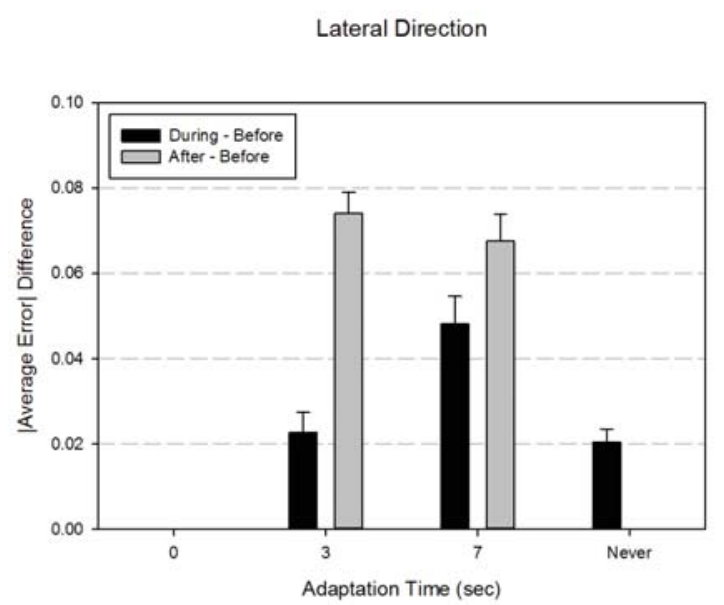

(a) Absolute Average Error Difference by Adaptation Time and Section for Lateral Direction

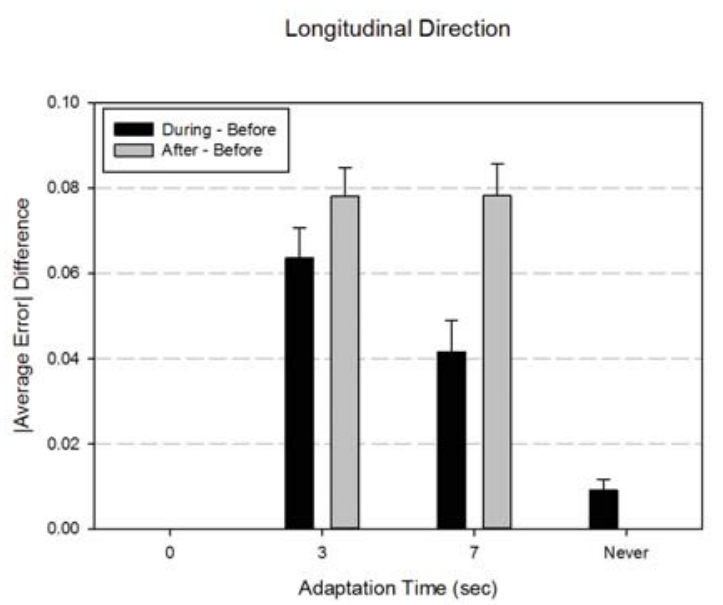

(b) Absolute Average Error Difference by Adaptation Time and Section for Longitudinal Direction

Figure 4. Absolute Average Error Difference Interaction

\section{Parameter Value Difference Between Sections}

When looking at the differences in parameter values during and after from before section parameter values (i.e, during-before and after-before sections), there was an algorithm by direction and section interaction $\left(F_{(3,4615)}=4.89, p \leq 0.01\right)$. As can be seen in Figure 6 on page 9, the GDE algorithm has the most difference in parameter values for the during - before and after - before sections. There were also an adaptation time and direction, and adaptation time and algorithm interaction with $\mathrm{PV}_{[d b, a b]}\left(F_{(2,5615)}=6.63, p \leq 0.01\right.$ and $F_{(4,5615)}=8.08, p \leq 0.01$ respectively). As seen in Figure 7(a) on page 9, the lateral direction had the most change in $\mathrm{PV}$ for both during - before and after - before sections. The GDE algorithm also has 


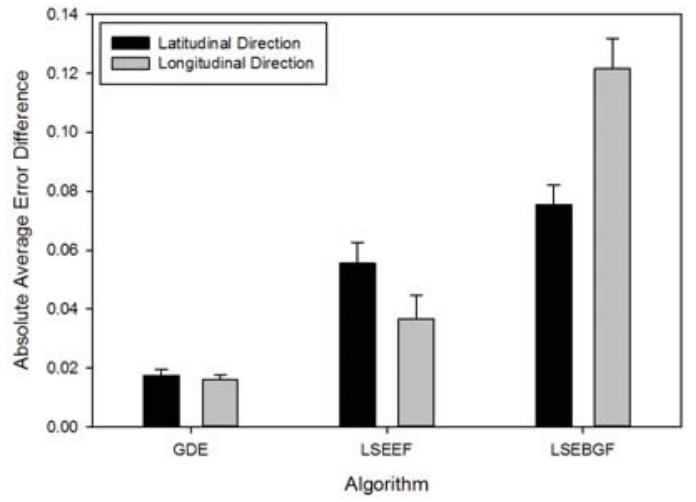

(a) Absolute Average Error Difference by Algorithm and Direction

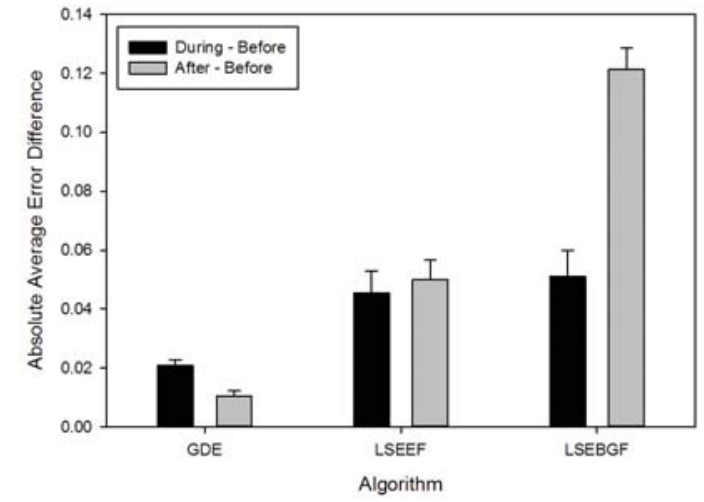

(b) Absolute Average Error Difference by Algorithm and Section

Figure 5. Algorithm Effects on Absolute Average Error Difference

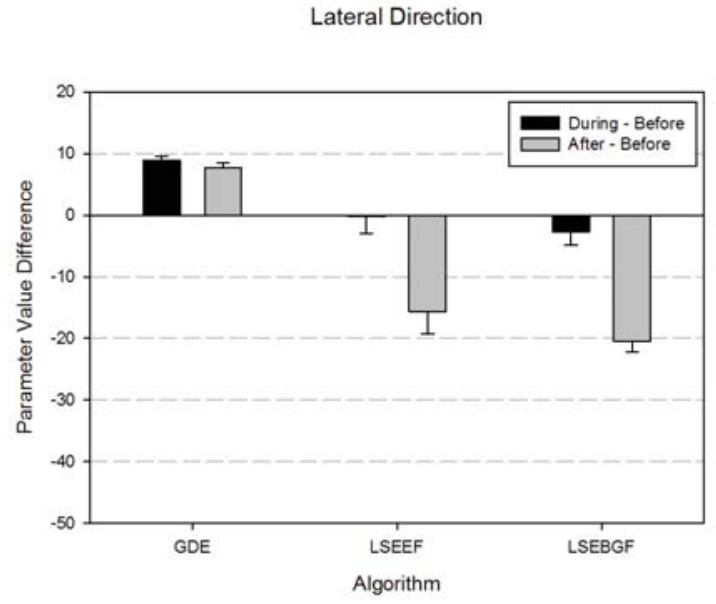

(a) Parameter Value Difference by Algorithm for Lateral Direction

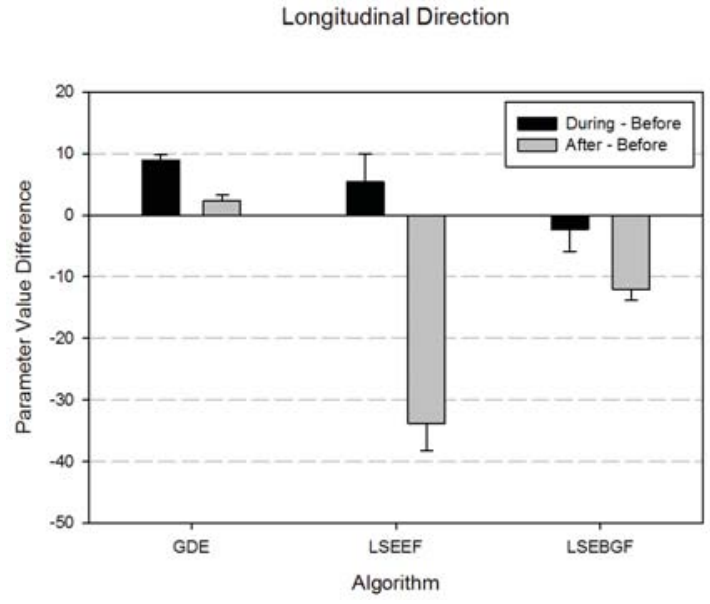

(b) Parameter Value Difference by Algorithm for Longitudinal Direction

Figure 6. Algorithm Effects on Parameter Value Difference by Direction

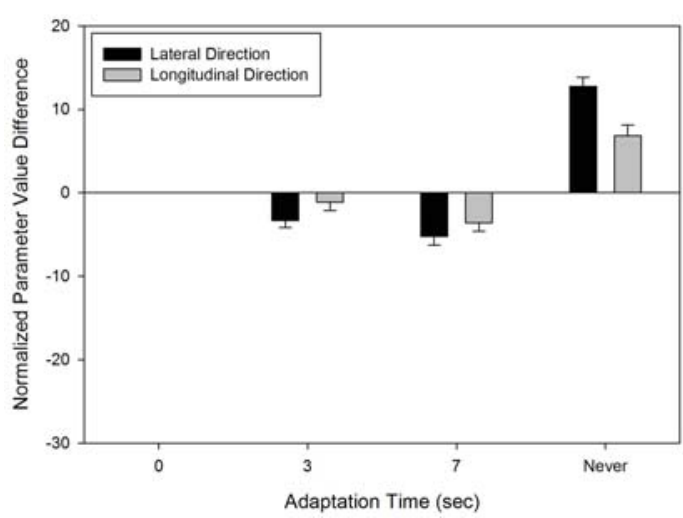

(a) Parameter Value Difference by Direction and Adaptation Time

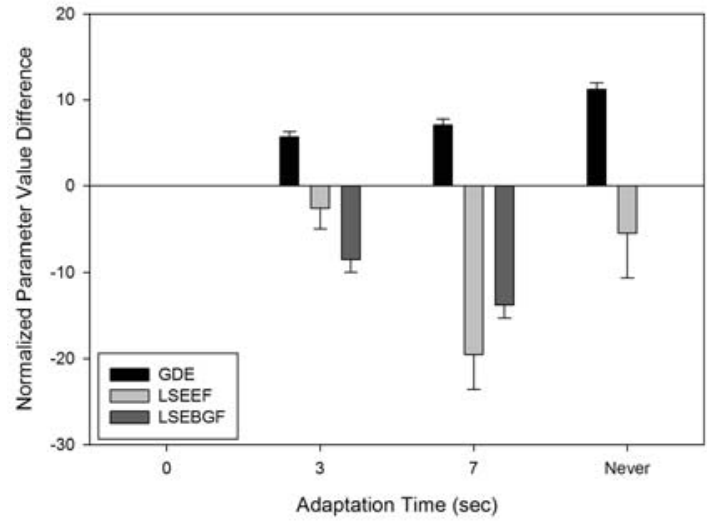

(b) Parameter Value Difference by Algorithm and Adaptation Time

Figure 7. Adaptation Time Effects on Parameter Value Difference 
fairly large differences in parameter value for all adaptation times (Figure 7(b) on page 9). Therefore, when looking at changes in PV, the GDE algorithm in the lateral direction may best indicate changes in piloting methods.

\section{Conclusions}

In the last two decades, there has been a renewed interest in modeling the human operator. There has also been significant work on techniques used to identify the pilot model of a given structure. The purpose of this research is to attempt to go beyond pilot identification based on collected experimental data and to develop a predictor of pilot behavior. An experiment was conducted to categorize these interactions on the pilot with an adaptive controller during control surface failures. GDE, LSEEF, and LSEBGF algorithms used these data to predict pilot stick input.

The three algorithms were able to predict subject stick input with varying degrees of success. In general the GDE and LSEBGF algorithms appear to be the best methods to use to converge to a solution especially if the sampling times are short or if there is not much stick movement, such as during straight and level flight.

As for individual differences, the subjects in this experiment appeared to essentially separate into two groups - one with lower PVs and |Average Errors| and another with slightly higher PVs and |Average Errors|, indicating that some tuning to individuals may be of benefit in order to more accurately predict stick input. However, individual differences become a non-factor when comparing the differences between the PV and |Average Error| at different times. Therefore, using a standard baseline PV and |Average Error| to compare to values acquired during flight may be sufficient to detect piloting changes. Furthermore, the sampling time should be at least 3 and 7 seconds in order to detect a change. If it is shorter than 3 seconds, the change may not be sufficient enough to detect. However, if it is too long, the pilot may have time to adapt to the conditions such that any differences may be essentially nulled out.

When looking individually at the prediction of pilot stick input, the GDE algorithm and lateral direction movements were the least sensitive to subject effects as long as conditions were not changing significantly and the sampling times were short enough such that errors did not build up in the algorithm. Thus, if the PV is not specifically tuned to the pilot, then the GDE method in the lateral direction provides the most accuracy in predicting pilot stick input during normal flight operations with short sampling times. However, for longer runs or where there are constant changes in flight path, then the LSEBGF may be a better algorithm to estimate pilot stick input and to detect changes in piloting because of its forgetting characteristic.

If the goal is to strictly detect piloting changes, then focusing on the difference between baseline values and current values should suffice. In this case, the LSEBGF algorithm may be best for detecting changes in piloting behavior when looking at the absolute average error differences between sections. However, if changes in PV are focused on, then the GDE algorithm in the lateral direction may best indicate changes in piloting.

In summary, the GDE and LSEBGF algorithms have the potential to accurately predict pilot stick input from flight director commands. With this capability to predict pilot actions comes the ability to detect unexpected changes. Once these changes are detected, then additional data and further analyses may determine whether these changes are due to the pilot or a non-normal aircraft. If it is due to a non-normal aircraft, then on-board automation may be able to aid the pilot in determining the changing capabilities of the aircraft and to try to help alleviate any issues such as by automatically completing electronic checklists. If it is due to the pilot, then on-board automation may be able to aid the pilot by changing workload, focusing attention appropriately, or, as in the case with an incapacitated pilot, safely land the aircraft. Thus, this methodology may lead to a safer national airspace system.

\section{Acknowledgements}

The authors would like to thank John Foster and Kevin Cunningham of NASA Langley Research Center for their help in tuning the aircraft simulation model for the experiment described above. For insight into the estimation methods, the authors would like to thank Dr. Eugene Lavretsky, Senior Technical Fellow at Boeing.

This research was funded by NASA's Aviation Safety Program - Vehicle Systems Safety Technologies project and NASA Langley's Autonomy Incubator. 


\section{References}

${ }^{1}$ Bailey, R. and Mook, D. J., "Pilot Control Identification Using Minimum Model Error Identification," AIAA paper 92-1121, 1992

${ }^{2}$ Davidson, J. D. and Schmidt, D. K., "Modified Optimal Control Pilot Model for Computer-Aided Design and Analysis," NASA TM-4384, 1992.

${ }^{3}$ Doman, D. B. and Anderson, M. R., "A Fixed-Order Optimal Control Model of Human Operator Response," Automatica, Vol. 36, No. 3, 2000, pp. 409-418.

${ }^{4}$ Hess, R. A., "Obtaining Multi-Loop Pursuit-Control Pilot Models From Computer Simulation," AIAA paper 2007-247, 2007.

${ }^{5}$ Hess, R. A. and Mnich, M. A., "Identification of Pilot Dynamics from In-Flight Tracking Data," Proceedings of the AIAA Guidance, Navigation and Control Conference, AIAA, Snowmass, CO, Aug. 1985, pp. 554-563.

${ }^{6}$ McRuer, D. T. and Jex, H. R., "A Review of Quasi-Linear Pilot Models," IEEE Transactions on Human Factors in Electronics, Vol. HFE8, No. 3, 1967, pp. 231-249.

${ }^{7}$ McRuer, D. T. and Krendel, E. S., "Mathematical Models of Human Pilot Behavior," Tech. rep., 1974.

${ }^{8}$ Kleinman, D. L., Baron, S., and Levison, W. H., "An Optimal Control Model of Human Response - Part 1: Theory and Validation," Automatica, Vol. 6, No. 3, 1970, pp. 357-369.

${ }^{9}$ Hess, R. A., "Unified Theory for Aircraft Handling Qualities and Adverse Aircraft-Pilot Coupling," J. Guidance, Control, and Dynamics, Vol. 20, 1997, pp. 1141-1148.

${ }^{10}$ Schmidt, D. K., "Optimal Flight Control Synthesis Via Pilot Modeling," J. Guidance E Control, Vol. 2, No. 4, 1979, pp. 308-312.

${ }^{11}$ Hess, R. A., "Simplified Approach for Modeling Pilot Pursuit Control Behavior in Multi-Loop Flight Control Tasks," Journal of Aerospace Engineering, Vol. 220, No. G2, 2006, pp. 85-102.

${ }^{12}$ Hess, R. A., "A Preliminary Study of Human Pilot Dynamics in the Control of Time-Varying Systems," Proceedings of the AIAA Modeling and Simulation Conference, AIAA, Portland, OR, Aug. 2011.

${ }^{13}$ Brieger, O., Ossmann, D., and Müller, M., "A New Flight Test Technique for Pilot Model Identification," Proceedings of the AIAA Atmospheric Flight Mechanics Conference and Exhibit, AIAA, Honolulu, HI, Aug. 2008.

${ }^{14}$ Lampton, A. and Klyde, D., "Power Frequency - A New Metric for Analyzing Pilot-in-the-Loop Flying Tasks," Proceedings of the AIAA Atmospheric Flight Mechanics Conference and Exhibit, AIAA, Portland, OR, Aug. 2011.

${ }^{15}$ Thompson, P., Bachelder, E., Klyde, D., Harris, C., and Brenner, M., "Wavelet-Based techniques for Improved Online Systems Identification," Proceedings of the USAF Developmental Test and Evaluation Summit, AIAA, Woodland Hills, CA, Nov. 2004.

${ }^{16}$ Thompson, P., Klyde, D., and Brenner, M., "Wavelet-Based Time-Varying Human Operator Models," Proceedings of the AIAA Atmospheric Flight Mechanics Conference and Exhibit, AIAA, Montreal, Canada, Aug. 2001.

${ }^{17}$ Zaal, P., Pool, D. M., Mulder, M., and van Paassen, M. M., "Multimodal Pilot Control Behaviour in Combined TargetFollowing Disturbance-Rejection Tasks," Proceedings of the AIAA Modeling and Simulation Conference, AIAA, Chicago, IL, Aug. 2009.

${ }^{18}$ Zaal, P., Pool, D. M., Mulder, M., and van Paassen, M. M., "Multimodal Pilot Model Identification in Real Flight," Proceedings of the AIAA Modeling and Simulation Conference, AIAA, Chicago, IL, Aug. 2009.

${ }^{19}$ Zaal, P. and Sweet, B., "Estimation of Time-Varying Pilot Model Parameters," Proceedings of the AIAA Modeling and Simulation Conference, AIAA, Portland, OR, Aug. 2011.

${ }^{20}$ Trujillo, A. C. and Gregory, I. M., "Adaptive Controller Adaptation Time and Available Control Authority Affects on Piloting," Proceedings of the $1^{\text {tht }}$ International Symposium on Aviation Psychology, ISAP, Dayton, OH, May 2013.

${ }^{21}$ Trujillo, A. C. and Gregory, I. M., "Pilot Preferences on Displayed Aircraft Control Variables," Proceedings of the HCI International 2013 Conference, Las Vegas, NV, July 2013.

${ }^{22}$ Trujillo, A. C., Gregory, I. M., and Hempley, L., "Adaptive Controller Effects on Pilot Behavior," Proceedings of the IEEE 2014 International Conference on Systems, Man, and Cybernetics, San Diego, CA, Oct. 2014.

${ }^{23}$ Lavretsky, E., "System Identification (ID): CDS 270-1," class notes, California Institute of Technology, Pasadena, CA, 2008.

${ }^{24}$ Trujillo, A. C. and Gregory, I. M., "Preliminary Exploration of Adaptive State Predictor Based Human Operator Modeling," Proceedings of the 2012 AIAA Modeling and Simulation Technologies Conference, Minneapolis, MN, Aug. 2012.

${ }^{25}$ Trujillo, A. C. and Gregory, I. M., "Wetware, Hardware, or Software Incapacitation: Observational Methods to Determine When Autonomy Should Assume Control," Proceedings of the Aviation 2014 Conference, Atlanta, GA, June 2014. 